\title{
Milewicz, Karolina M. (2020): Constitutionalizing World Politics. The Logic of Democratic Power and the Unintended Consequences of International Treaty Making
}

\author{
Cambridge: Cambridge University Press. 354 Seiten. \$99.99
}

\section{Nina Reiners $\mathbb{D}$}

Angenommen: 29. Juni 2021 / Online publiziert: 19. Juli 2021

(C) Der/die Autor(en) 2021

Wie steht es um den Prozess der Konstitutionalisierung von Weltpolitik? Die Frage nach der Herausbildung einer Verfassung jenseits des Staates beleuchtet Karolina M. Milewicz in ihrer Monografie mit Blick auf die Staaten, die sich um dieses Ideal aufgrund ihrer innerstaatlichen Ordnung bemühen (,,willingness“") und die dazu auch die notwendigen Kapazitäten besitzen (,,ability“): mächtige Demokratien. Darin liegt für Milewicz die „logic of democratic power“: Wenn innerstaatlich Demokratie und Macht zusammentreffen, dann erhöht dies ebenfalls die ,willingness“ und ,ability“ zur Kooperation auf der internationalen Ebene und mächtige Demokratien werden so ,the strongest promoters of international treaty rules“ (S. 8). Ihr zentrales Argument lautet, dass das Ideal einer internationalen Verfassungsordnung zwar noch weit entfernt ist, aber durch mächtige Demokratien entscheidend vorangebracht wird wenn auch weitestgehend unbeabsichtigt. Durch eine ganze Reihe an vergleichenden Analysen zu formellen Regeln auf der internationalen Ebene und innerhalb westlicher Demokratien leistet die Studie einen wichtigen Beitrag zur Verfassungsliteratur in den Internationalen Beziehungen und der Vergleichenden Politikwissenschaft. Das Buch dürfte darüber hinaus für Leser*innen mit Interesse an Demokratietheorie relevant sein. Doch ist, wie der Untertitel des Buches suggeriert, die Konstitutionalisierung von Weltpolitik wirklich eine unbeabsichtigte Konsequenz internationaler Verträge? In meiner Rezension werde ich mich deshalb schwerpunktmäßig dem Befund von Milewicz zuwenden, dass „Constitutionalizing World Politics“ als Prozess $\mathrm{zu}$ verstehen ist, der von Staaten bei der Schaffung von internationalem Recht nicht beabsichtigt wird.

Der Blick auf das Cover des Buches gibt zunächst einen Überblick über die historische Entwicklung des Konstitutionalisierungsprozesses. Die Autorin hat sich für

Nina Reiners $(\varangle)$

Universität Potsdam, Potsdam, Deutschland

E-Mail: nreiners@uni-potsdam.de 
die Abbildung der Unterzeichnung der Magna Charta auf der einen Seite und dem breit rezipierten Foto des G-7-Gipfels von 2018 auf der anderen Seite entschieden. Die visuelle Verbindung zwischen dem englischen Adel, der King John zur Unterzeichnung der Magna Charta drängt, und den Staatschefs, die energisch auf den ehemaligen US-Präsidenten Trump einreden, setzt allerdings nicht etwa den Rahmen für den Untersuchungszeitraum, sondern verdeutlicht das Argument der Autorin am Ende ihrer detaillierten empirischen Studie. Für Milewicz zeigt sich in beiden Fällen, dass auch kurzfristige Rückschläge und Skepsis im Prozess globaler Kooperation Quellen für langfristigen Optimismus sein können (S. 240).

Dieses Fazit hat seinen Ausgangspunkt in der Beobachtung, dass die Literatur zur Konstitutionalisierung der Weltpolitik in zwei Lager geteilt ist: Auf der einen Seite sieht Milewicz die optimistischen Idealist*innen, die sie vorwiegend innerhalb der Rechtswissenschaft verortet. Auf der anderen Seite stehen für sie die pessimistischen Realist*innen, die mit ihrer Betonung von Machtpolitik die Möglichkeit von Kooperation gänzlich ausschließen. Die Autorin argumentiert für einen realistischskeptischen Blick auf die Möglichkeiten internationaler Konstitutionalisierung und verortet ihr Argument als ,middle position“ (S. 2) zwischen Idealist*innen und Realist*innen. Für die Verdeutlichung ihres Arguments ist die Zuspitzung der beiden Positionen nachvollziehbar, jedoch wäre eine differenzierte Diskussion der rechtsund politikwissenschaftlichen Forschung zu diesem Thema durchaus wünschenswert gewesen.

Der polarisierende Blick auf die Literatur ergibt sich auch aus dem Interesse an ihrem empirischen Untersuchungsgegenstand. Milewicz nimmt dafür formelle zwischenstaatliche Verträge in den Blick. Sie widmet sich in ihren Analysen einem Sample von 75 internationalen Verträgen, die seit 1945 ratifiziert wurden. Dieser Fokus wird von ihr überaus nachvollziehbar begründet. Dennoch ist der gewählte Fokus auf die Ratifikation von Verträgen nur ein Teil der umfassend beleuchteten Frage, wie Recht und (internationale) Verträge Wirkung auf das Verhalten von Staaten ausüben können - ob von diesen beabsichtigt oder nicht. Die Ergebnisse ihrer hervorragenden statistischen Analysen zur „logic of democratic power" in den Phasen der Verhandlung, Ratifizierung und der Einhaltung von Verträgen (Kapitel 6) können durchaus auch zu weiteren Fragen der (globalen) Verfassungstheorie einen empirischen Beitrag leisten und lassen sich mit Gewinn diskutieren: Wenn sich, wie Milewicz betont, der Konstitutionalisierungsprozess völlig unterschiedlich in einzelnen Politikfeldern gestaltet, was bringt diese dann als eine Verfassungsordnung zusammen? Welche Rolle spielen internationale Regeln für den (unbeabsichtigten) globalen Konstitutionalisierungsprozess, die nicht in Form von Verträgen ratifiziert werden? So sieht Milewicz beispielsweise im Bereich der Menschenrechte den „Internationalen Pakt über zivile und politische Rechte“ (Zivilpakt) als Bestätigung des Mechanismus der Logik demokratischer Macht. Dennoch hat auch die ,Allgemeine Erklärung der Menschenrechte“ von 1948 längst den Status von Gewohnheitsrecht erlangt, auch durch ihre Aufnahme in zahlreiche nationale Verfassungen von weniger mächtigen und stabilen Demokratien. Aus der Annahme dieser unverbindlichen Erklärung entwickelte sich innerhalb weniger Dekaden ein universelles Vertragssystem zum internationalen Menschenrechtsschutz. 
Für Milewicz wäre diese Entwicklung von den beteiligten (mächtigen) Demokratien nicht beabsichtigt gewesen, da sie die Verhandlungen von einzelnen Menschenrechtsverträgen isoliert von anderen Entwicklungen betrachtet. Ihre Ausführungen zur erfolgreichen Durchsetzung demokratischer Delegationen bei den Verhandlungen des Zivilpaktes (S. 205-207) erfolgen zum Beispiel gänzlich ohne Verweis auf den zeitgleich ebenfalls verabschiedeten ,Internationalen Pakt über wirtschaftliche, soziale und kulturelle Rechte“ (Sozialpakt) - welcher genauso als Nichterfolg mächtiger Demokratien interpretiert werden kann. Die Entwicklungen hin zu einem universellen Menschenrechtsvertragssystem, wie wir es heute vorfinden, kann eben nicht nur mit Verweis auf mächtige Demokratien erklärt werden. Aus einer AgencyPerspektive wäre hier zum einen die Ausweitung des Blicks auch auf nichtstaatliche Akteur*innen wichtig gewesen, die Konstitutionalisierung jenseits des Staates durchaus im Blick haben. Aus einer stärker historischen Perspektive betrachtet finden sich Treiber einer hierarchischen Ordnung auch in anderen Regionen und auf allen Ebenen der Weltpolitik. Zum anderen ließe sich das Argument der ,unintended consequences of international treaty-making“ hervorragend auf Akteure ausweiten, die seit 1945 von Staaten zur Überwachung der Einhaltung dieser Verträge eingesetzt wurden und z. B. durch Interpretationen und Entscheidungen von Richter*innen und Expert*innenausschüssen die Entwicklung von internationalem Recht vorantreiben.

Das Buch lädt somit angesichts der weltweiten Abnahme der Qualität von Demokratien in den letzten zehn Jahren dazu ein, über das Projekt der Konstitutionalisierung von Weltpolitik mit Blick auf ihre Unterstützer*innen nachzudenken. Milewicz realistisch-skeptischer Blick wird auch mit Blick auf die Herausbildung konstitutioneller Ordnungen ohne demokratische Autorisierung, so etwa den autoritären Konstitutionalismus, kommende Analysen gewinnbringend informieren. Sozialisierungsprozesse verlaufen eben nicht nur in eine Richtung. Milewicz größtes Verdienst ist es zweifellos, dass sie mit dieser Studie detaillierte und methodisch anspruchsvolle Analysen der nationalen und der internationalen Ebene in einem klaren Argument auch für ein breiteres Publikum zugänglich macht. Schaut man nach der Lektüre wieder auf das Cover, so ist man um wesentliche Kenntnisse der Elemente des Konstitutionalismus sowie empirische Befunde der jüngeren Demokratie- und Verfassungspolitik reicher.

Funding Open Access funding enabled and organized by Projekt DEAL.

Open Access Dieser Artikel wird unter der Creative Commons Namensnennung 4.0 International Lizenz veröffentlicht, welche die Nutzung, Vervielfältigung, Bearbeitung, Verbreitung und Wiedergabe in jeglichem Medium und Format erlaubt, sofern Sie den/die ursprünglichen Autor(en) und die Quelle ordnungsgemäß nennen, einen Link zur Creative Commons Lizenz beifügen und angeben, ob Änderungen vorgenommen wurden.

Die in diesem Artikel enthaltenen Bilder und sonstiges Drittmaterial unterliegen ebenfalls der genannten Creative Commons Lizenz, sofern sich aus der Abbildungslegende nichts anderes ergibt. Sofern das betreffende Material nicht unter der genannten Creative Commons Lizenz steht und die betreffende Handlung nicht nach gesetzlichen Vorschriften erlaubt ist, ist für die oben aufgeführten Weiterverwendungen des Materials die Einwilligung des jeweiligen Rechteinhabers einzuholen.

Weitere Details zur Lizenz entnehmen Sie bitte der Lizenzinformation auf http://creativecommons.org/ licenses/by/4.0/deed.de. 